\title{
Violation of all two-party facet Bell inequalities by almost-quantum correlations
}

\author{
Ravishankar Ramanathan $0^{*}$ \\ Department of Computer Science, The University of Hong Kong, Pokfulam Road, Hong Kong
}

(Received 15 June 2020; accepted 21 June 2021; published 29 July 2021)

\begin{abstract}
The characterization of the set of quantum correlations is a problem of fundamental importance in quantum information. The question whether every proper (tight) Bell inequality is violated in quantum theory is an intriguing one in this regard. Here we make significant progress in answering this question, by showing that every tight Bell inequality is violated by "almost-quantum" correlations, a semidefinite programming relaxation of the set of quantum correlations. As a consequence, we show that many (classes of) Bell inequalities including two-party correlation Bell inequalities and multioutcome nonlocal computation games, which do not admit quantum violations, are not facets of the classical Bell polytope. To do this, we make use of the intriguing connections between Bell correlations and the graph-theoretic Lovász-theta set, discovered by Cabello-SeveriniWinter (CSW). We also exploit connections between the cut polytope of graph theory and the classical correlation Bell polytope, to show that correlation Bell inequalities that define facets of the lower dimensional correlation polytope are violated in quantum theory.
\end{abstract}

DOI: 10.1103/PhysRevResearch.3.033100

Quantum correlations, i.e., the correlations between quantum systems in a Bell-type experiment, are of central interest in quantum information theory. Their violation of Bell inequalities shows, in a device-independent manner, that quantum theory fundamentally differs from all classical theories. These quantum "nonlocal" correlations also allow us to perform tasks that are impossible in classical theory, such as generation of cryptographic key secure against post-quantum eavesdroppers $[1,2]$, intrinsic randomness certification and amplification [3-6], and reduction of communication complexity [7]. For fundamental reasons as well as to develop these applications, it is of utmost importance to characterize the set of quantum correlations, and understand how it fits in between the polytopes of classical and general nonsignaling correlations [8-10].

The proper (tight) Bell inequalities are those that define facets of the classical polytope, while not also defining facets of the no-signaling polytope [8-10]. A problem of fundamental importance in the characterization of quantum correlations was raised by Gill in [11], namely whether every tight Bell inequality is violated in quantum theory. The analogous question pertaining to facets of the binary-outcome correlation polytope (the classical polytope of two-party binary-outcome correlations, excluding the local marginal terms) was raised by Avis et al. in [12]. Escolá, Calsamiglia, and Winter in a recent breakthrough result [13] answered the latter question, showing that the binary-outcome correlation polytope does

\footnotetext{
*ravi@cs.hku.hk

Published by the American Physical Society under the terms of the Creative Commons Attribution 4.0 International license. Further distribution of this work must maintain attribution to the author(s) and the published article's title, journal citation, and DOI.
}

not share any nontrivial facet-defining inequalities (understood as inequalities defining a face of dimension one less than the set) in common with the set of quantum correlations. The corresponding question for multiparty tight Bell inequalities had been previously answered in a fundamental breakthrough result by Fritz et al. [14] who identified a class of nontrivial tight Bell inequalities (called local orthogonality inequalities) that are not violated in quantum theory following results in $[15,16]$, when three or more parties are involved in the Bell experiment. The corresponding local orthogonality principle is a fundamental information-theoretic principle that serves to delineate the set of correlations realizable in a physical theory. In the multipartite Bell scenario, it only remains an open question whether all nontrivial facet Bell inequalities without quantum violation are of the local orthogonality form. In the bipartite Bell experiment though, the local orthogonality constraints reduce to the no-signaling conditions, and do not provide any nontrivial facet constraints to the quantum correlation set.

In this paper we study the question of whether there are tight two-party Bell inequalities with no quantum violation. We first describe novel classes of two-party Bell inequalities that do not admit quantum violation, including certain two-party correlation Bell inequalities and their multioutcome generalization. We then prove our central result that all two-party Bell inequalities that define facets of the classical Bell polytope are violated by a natural semidefinite programming relaxation to the set of quantum correlations that has been dubbed almost-quantum theory. We show how the novel classes introduced earlier are proven to not describe facets as a consequence of the result, as well as how it subsumes a recent breakthrough on two-party XOR games with no quantum advantage. Finally, we also show how connections discovered by Avis et al. in [12] can be used to show that all correlation 
Bell inequalities that define facets of the lower dimensional correlation Bell polytope are violated in quantum theory. We end with a brief discussion and open questions.

\section{PRELIMINARIES}

Consider a two-party Bell experiment. Suppose one party Alice chooses to measure one of $m_{A}$ inputs $i_{A}=1, \ldots, m_{A}$, and obtains one of $d_{A, i_{A}}$ outputs $o_{A} \in\left\{1, \ldots, d_{A, i_{A}}\right\}$. Similarly, the other party Bob chooses to measure one of $m_{B}$ inputs $i_{B}=$ $1, \ldots, m_{B}$, and obtains one of $d_{B, i_{B}}$ outputs $o_{B} \in\left\{1, \ldots, d_{B, i_{B}}\right\}$ outputs. Such a Bell scenario is denoted by the notation $\mathbf{B}\left(2 ; m_{A}, \vec{d}_{A} ; m_{B}, \vec{d}_{B}\right)$, where $\vec{d}_{A}=\left(d_{A, 1}, \ldots, d_{A, m_{A}}\right)$ and $\vec{d}_{B}=$ $\left(d_{B, 1}, \ldots, d_{B, m_{B}}\right)$. In some instances, this notation can also be shortened to $\mathbf{B}\left(\vec{d}_{A}, \vec{d}_{B}\right)$ for simplicity. The joint probability of obtaining the outcomes $\left(o_{A}, o_{B}\right)$ given the measurement settings $\left(i_{A}, i_{B}\right)$ is denoted as $P_{O_{A}, O_{B} \mid I_{A}, I_{B}}\left(o_{A}, o_{B} \mid i_{A}, i_{B}\right)$. We will view these $n=\left(\sum_{i_{A}=1}^{m_{A}} d_{A, i_{A}}\right)\left(\sum_{i_{B}=1}^{m_{B}} d_{B, i_{B}}\right)$ probabilities as forming the components of a vector $P_{O_{A}, O_{B} \mid I_{A}, I_{B}}=|P\rangle$ in $\mathbb{R}^{n}$, where the inputs and outputs are implicit, and the probabilities are also described as forming a box $P$.
In the Bell scenario $\mathbf{B}((2,2),(2,2))$ where each party chooses two dichotomic observables, all the facet inequalities are known: up to permutation of the outcomes they correspond to the well-known Clauser-Horne-Shimony-Holt inequality [17]. While it is in principle possible using specific facet-enumeration algorithms to obtain all the facet inequalities of the classical polytope in any given Bell scenario, in practice the complexity of the problem grows rapidly and facet inequalities have been found in cases with a few more observables and outcomes [18]. In fact, the problem of listing all facet Bell inequalities has been demonstrated to be NP-complete [19] making this an important but hard-to-solve problem in the theory of quantum nonlocality.

The box $P$ is a valid normalized no-signaling box, satisfying the no-signaling constraints of relativity and the normalization of probabilities, if it obeys the constraints of

(1) Non-negativity: $P_{O_{A}, O_{B} \mid I_{A}, I_{B}}\left(o_{A}, o_{B} \mid i_{A}, i_{B}\right) \geqslant 0$ for all $o_{A}, o_{B}, i_{A}, i_{B}$,

(2) Normalization: $\sum_{o_{A}=1}^{d_{A, i_{A}}} \sum_{o_{B}=1}^{d_{B, i_{B}}} P_{O_{A}, O_{B} \mid I_{A}, I_{B}}\left(o_{A}, o_{B} \mid i_{A}, i_{B}\right)=$ 1 for all $i_{A}, i_{B}$

(3) No-signaling:

$$
\begin{aligned}
& \sum_{o_{A}=1}^{d_{A, i_{A}}} P_{O_{A}, O_{B} \mid I_{A}, I_{B}}\left(o_{A}, o_{B} \mid i_{A}, i_{B}\right)=\sum_{o_{A}^{\prime}=1}^{d_{A, i_{A}^{\prime}}} P_{O_{A}, O_{B} \mid I_{A}, I_{B}}\left(o_{A}^{\prime}, o_{B} \mid i_{A}^{\prime}, i_{B}\right) \text { for all } i_{A}, i_{A}^{\prime}, o_{B}, i_{B}, \\
& \sum_{o_{B}=1}^{d_{B, i_{B}}} P_{O_{A}, O_{B} \mid I_{A}, I_{B}}\left(o_{A}, o_{B} \mid i_{A}, i_{B}\right)=\sum_{o_{B}^{\prime}=1}^{d_{B, i_{B}^{\prime}}} P_{O_{A}, O_{B} \mid I_{A}, I_{B}}\left(o_{A}, o_{B}^{\prime} \mid i_{A}, i_{B}^{\prime}\right) \text { for all } i_{B}, i_{B}^{\prime}, o_{A}, i_{A} .
\end{aligned}
$$

The boxes $P$ that satisfy the above constraints form the no-signaling polytope of the Bell scenario $\mathbf{N S}\left[\mathbf{B}\left(2 ; m_{A}, \vec{d}_{A} ; m_{B}, \vec{d}_{B}\right)\right]$. A fundamental result in polyhedral theory, known as the Minkowski-Weyl theorem, states that a polytope represented as the convex hull of a finite number of points, such as $\mathbf{N S}\left[\mathbf{B}\left(2 ; m_{A}, \vec{d}_{A} ; m_{B}, \vec{d}_{B}\right)\right]$ can also be equivalently represented as the intersection of finitely many half-spaces. One may write the above constraints in the form of inequalities, with the normalization and no-signaling equalities being written as two inequalities, and rewrite the no-signaling polytope in the following canonical form:

$$
\mathbf{N S}\left[\mathbf{B}\left(2 ; m_{A}, \vec{d}_{A} ; m_{B}, \vec{d}_{B}\right)\right]=\left\{|P\rangle \in \mathbb{R}^{n}: A \cdot|P\rangle \leqslant|b\rangle\right\}
$$

Here the matrix $A$ is an $m \times n$ matrix, with $m=$ $n+2 m_{A} m_{B}+2\left(m_{A}-1\right) \sum_{i_{B}=1}^{m_{B}}\left(d_{B, i_{B}}-1\right)+2\left(m_{B}-\right.$ 1) $\sum_{i_{A}=1}^{m_{A}}\left(d_{A, i_{A}}-1\right)$. This value for $m$ comes from $n$ non-negativity constraints, $m_{A} m_{B}$ normalization equalities, and $\left(m_{A}-1\right) \sum_{i_{B}=1}^{m_{B}}\left(d_{B, i_{B}}-1\right)$ no-signaling equalities defining Bob's marginal probabilities and similarly $\left(m_{B}-1\right) \sum_{i_{A}=1}^{m_{A}}\left(d_{A, i_{A}}-1\right)$ no-signaling equalities defining Alice's marginal probabilities. The vector $|b\rangle$ is an appropriate defined $m$-dimensional vector with entries in $\{0,1,-1\}$. Crucially, this gives the dimensionality of the no-signaling polytope to be

$$
\begin{aligned}
\operatorname{dim}(\mathbf{N S} & {\left.\left[\mathbf{B}\left(2 ; m_{A}, \vec{d}_{A} ; m_{B}, \vec{d}_{B}\right)\right]\right) } \\
= & \left(\sum_{i_{A}=1}^{m_{A}}\left(d_{A, i_{A}}-1\right)+1\right) \\
& \times\left(\sum_{i_{B}=1}^{m_{B}}\left(d_{B, i_{B}}-1\right)+1\right)-1=: D .
\end{aligned}
$$

The boxes within the no-signaling polytope that additionally satisfy the integrality constraint given by

(4) Integrality $P_{O_{A}, O_{B} \mid I_{A}, I_{B}}\left(o_{A}, o_{B} \mid i_{A}, i_{B}\right) \in\{0,1\}$ for all $o_{A}, o_{B}, i_{A}, i_{B}$,

are said to be local deterministic boxes (LDBs). The convex hull of these LDBs forms the classical or Bell polytope denoted by $\mathbf{C}\left[\mathbf{B}\left(2 ; m_{A}, \vec{d}_{A} ; m_{B}, \vec{d}_{B}\right)\right]$. This is the set of all correlations obtainable from local hidden variable theories.

The set of quantum correlations denoted by $\mathbf{Q}\left[\mathbf{B}\left(2 ; m_{A}, \vec{d}_{A} ; m_{B}, \vec{d}_{B}\right)\right]$ also lies within the no-signaling polytope. This set consists of boxes $P$ where each component $P_{O_{A}, O_{B} \mid I_{A}, I_{B}}\left(o_{A}, o_{B} \mid i_{A}, i_{B}\right)$ is obtained as

$$
P_{O_{A}, O_{B} \mid I_{A}, I_{B}}\left(o_{A}, o_{B} \mid i_{A}, i_{B}\right)=\operatorname{Tr}\left[\rho\left(E_{i_{A}, o_{A}}^{A} \otimes E_{i_{B}, o_{B}}^{B}\right)\right]
$$

for some quantum state $\rho \in \mathcal{H}_{d}$ of some arbitrary dimension $d$, and sets of projection operators $\left\{E_{i_{A}, o_{A}}^{A}\right\}$ for Alice and $\left\{E_{i_{B}, o_{B}}^{B}\right\}$ for Bob. Notably, the measurement 
operators satisfy the requirements of (i) Hermiticity: $\left(E_{i_{A}, o_{A}}^{A}\right)^{\dagger}=E_{i_{A}, o_{A}}^{A}$ for all $i_{A}, o_{A}$ and $\left(E_{i_{B}, o_{B}}^{B}\right)^{\dagger}=E_{i_{B}, o_{B}}^{B}$ for all $i_{B}, o_{B}$; (ii) orthogonality: $E_{i_{A}, o_{A}}^{A} E_{i_{A}, o_{A}^{\prime}}^{A}=\delta_{o_{A}, o_{A}^{\prime}} E_{i_{A}, o_{A}}^{A}$ for all $i_{A}$ and $E_{i_{B}, o_{B}}^{B} E_{i_{B}, o_{B}^{\prime}}^{B}=\delta_{o_{B}, o_{B}^{\prime}} E_{i_{B}, o_{B}}^{B}$ for all $i_{B}$; and (iii) completeness: $\sum_{o_{A}} E_{i_{A}, o_{A}}^{A}=\mathbb{1}$ for all $i_{A}$ and $\sum_{o_{B}} E_{i_{B}, o_{B}}^{B}=\mathbb{1}$ for all $i_{B}$. The set $\mathbf{Q}\left[\mathbf{B}\left(2 ; m_{A}, \vec{d}_{A} ; m_{B}, \vec{d}_{B}\right)\right]$ is convex but not a polytope. We have the inclusions $\mathbf{C}\left[\mathbf{B}\left(2 ; m_{A}, \vec{d}_{A} ; m_{B}, \vec{d}_{B}\right)\right] \subseteq$ $\mathbf{Q}\left[\mathbf{B}\left(2 ; m_{A}, \vec{d}_{A} ; m_{B}, \vec{d}_{B}\right)\right] \subseteq \mathbf{N S}\left[\mathbf{B}\left(2 ; m_{A}, \vec{d}_{A} ; m_{B}, \vec{d}_{B}\right)\right]$.

By the Minkowski-Weyl theorem, the set $\mathbf{C}\left[\mathbf{B}\left(2 ; m_{A}, \vec{d}_{A} ; m_{B}, \vec{d}_{B}\right)\right]$ can also be equivalently represented as the intersection of finitely many half-spaces

$$
\mathbf{C}\left[\mathbf{B}\left(2 ; m_{A}, \vec{d}_{A} ; m_{B}, \vec{d}_{B}\right)\right]=\left\{|P\rangle \in \mathbb{R}^{n}: B_{G_{i}} \cdot|P\rangle \leqslant \omega_{c}\left(G_{i}\right) \quad \forall i \in I\right\}
$$

where $\left\{B_{G_{i}} \cdot|P\rangle \leqslant \omega_{c}\left(G_{i}\right), \quad i \in I\right\}$ is a finite set of inequalities. The inequalities supporting facets of $\mathbf{C}\left[\mathbf{B}\left(2 ; m_{A}, \vec{d}_{A} ; m_{B}, \vec{d}_{B}\right)\right]$ provide a minimal set of such inequalities, and are usually referred to as facet Bell inequalities, or in some instances in the literature just as the Bell inequalities. In particular, any valid inequality for $\mathbf{C}\left[\mathbf{B}\left(2 ; m_{A}, \vec{d}_{A} ; m_{B}, \vec{d}_{B}\right)\right]$ can be derived from the facet inequalities.

The introduction of a few notions from polytope theory is in order here. Boxes $P_{1}, \ldots, P_{m}$ in $\mathbb{R}^{n}$ are said to be affinely independent if the unique solution to $\sum_{i=1}^{m} \mu_{i} P_{i}=0$, $\sum_{i=1}^{m} \mu_{i}=0$ is that $\mu_{i}=0$ for all $i=1, \ldots, m$. Equivalently, the boxes are affinely independent if $P_{2}-P_{1}, \ldots, P_{m}-P_{1}$ are linearly independent. The affine hull of a set of boxes is the set of all their affine combinations. The affine set has dimension $K$, if the maximum number of affinely independent boxes it contains is $K+1$. An inequality $B_{G_{i}} \cdot|P\rangle \leqslant \omega_{c}\left(G_{i}\right)$ satisfied by all boxes in $\mathbf{C}\left[\mathbf{B}\left(2 ; m_{A}, \vec{d}_{A} ; m_{B}, \vec{d}_{B}\right)\right]$ is called a valid Bell inequality. Given a valid inequality $B_{G_{i}} \cdot|P\rangle \leqslant \omega_{c}\left(G_{i}\right)$, the set

$$
F=\left\{|P\rangle \in \mathbb{R}^{n}: B_{G_{i}} \cdot|P\rangle=\omega_{c}\left(G_{i}\right)\right\}
$$

is called a face of the classical polytope and the inequality is said to support $F$. The dimension of $F$ is the dimension of its affine hull. If $F \neq \varnothing$ (the empty set) and $F \neq$ $\mathbf{C}\left[\mathbf{B}\left(2 ; m_{A}, \vec{d}_{A} ; m_{B}, \vec{d}_{B}\right)\right]$, it is a proper face. Proper faces satisfy by definition $\operatorname{dim}(F) \leqslant \operatorname{dim}\left(\mathbf{C}\left[\mathbf{B}\left(2 ; m_{A}, \vec{d}_{A} ; m_{B}, \vec{d}_{B}\right)\right]\right)-$ $1=D-1$. Proper faces of maximal dimension are called facets. A Bell inequality $B_{G_{i}} \cdot|P\rangle \leqslant \omega_{c}\left(G_{i}\right)$ thus supports a facet of the classical polytope if and only if $D$ affinely independent boxes of $\mathbf{C}\left[\mathbf{B}\left(2 ; m_{A}, \vec{d}_{A} ; m_{B}, \vec{d}_{B}\right)\right]$ satisfy it with equality.

Finding the quantum violation of a Bell inequality is also a well-known hard problem. In the special instance of twoparty correlation Bell inequalities, also known as XOR games, the quantum value can be directly determined by means of a semidefinite program, as shown by Tsirelson [20]. For more general two-party Bell inequalities, where the parties observe more than two measurement outcomes, or where the inequality also includes terms involving marginal probabilities observed by either party, finding the quantum violation is not as easy. In [21], a hierarchy of semidefinite programs was formulated for optimization with noncommuting variables, and this NPA hierarchy is ubiquitously employed to efficiently determine upper bounds to the quantum violation for general Bell inequalities. The hierarchy was also shown to converge to a set $\mathbf{Q}^{p r}\left[\mathbf{B}\left(2 ; m_{A}, \vec{d}_{A} ; m_{B}, \vec{d}_{B}\right)\right]$, which is the set consisting of boxes $P$ where each component $P_{O_{A}, O_{B} \mid I_{A}, I_{B}}\left(o_{A}, o_{B} \mid i_{A}, i_{B}\right)$ is obtained as

$$
P_{O_{A}, O_{B} \mid I_{A}, I_{B}}\left(o_{A}, o_{B} \mid i_{A}, i_{B}\right)=\operatorname{Tr}\left[\rho\left(E_{i_{A}, o_{A}}^{A} E_{i_{B}, o_{B}}^{B}\right)\right],
$$

with $\left[E_{i_{A}, o_{A}}^{A}, E_{i_{B}, o_{B}}^{B}\right]=0$ for all $i_{A}, o_{A}, i_{B}, o_{B}$. The above differs from Eq. (4) in that the strict requirement of tensor product structure is replaced with the requirement of only commutation between different parties' measurements. It is clear that $\mathbf{Q}\left[\mathbf{B}\left(2 ; m_{A}, \vec{d}_{A} ; m_{B}, \vec{d}_{B}\right)\right] \subseteq \mathbf{Q}^{p r}\left[\mathbf{B}\left(2 ; m_{A}, \vec{d}_{A} ; m_{B}, \vec{d}_{B}\right)\right]$.

In the NPA hierarchy, one considers sets consisting of sequences of product projection operators $S_{1}=\{\mathbb{1}\} \cup$ $\left\{E_{i_{A}, o_{A}}^{A}\right\} \cup\left\{E_{i_{B}, o_{B}}^{B}\right\}, S_{2}=S_{1} \cup\left\{E_{i_{A}, o_{A}}^{A} E_{i_{B}, o_{B}}^{B}\right\}$, etc. The convex sets corresponding to different levels of this hierarchy $\mathbf{Q}_{l}\left[\mathbf{B}\left(2 ; m_{A}, \vec{d}_{A} ; m_{B}, \vec{d}_{B}\right)\right]$ are constructed by testing for the existence of a certificate $\Gamma^{l}$ associated to the set of operators $S_{l}$ by means of a semidefinite program. This certificate $\Gamma^{l}$ corresponding to level $l$ of the NPA hierarchy is a $\left|S_{l}\right| \times$ $\left|S_{l}\right|$ matrix whose rows and columns are indexed by the operators in the set $S_{l}$. The certificate $\Gamma^{l}$ is required to be a complex Hermitian positive semidefinite matrix satisfying the following constraints on its entries: (i) $\Gamma_{\mathbb{1}, \mathbb{1}}^{l}=$ 1 and (ii) $\Gamma_{Q, R}^{l}=\Gamma_{S, T}^{l}$ if $Q^{\dagger} R=S^{\dagger} T$. The latter condi-

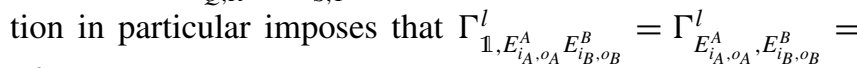

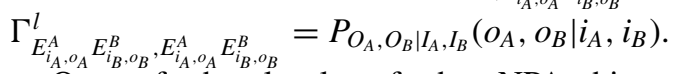

One of the levels of the NPA hierarchy denoted $\mathbf{Q}_{1+A B}\left[\mathbf{B}\left(2 ; m_{A}, \vec{d}_{A} ; m_{B}, \vec{d}_{B}\right)\right]$ or $\tilde{\mathbf{Q}}\left[\mathbf{B}\left(2 ; m_{A}, \vec{d}_{A} ; m_{B}, \vec{d}_{B}\right)\right]$ has been highlighted as being the almost-quantum set [22]. This set corresponds to an intermediate level of the hierarchy and is associated with the set of operators $\tilde{S}=\{\mathbb{1}\} \cup\left\{E_{i_{A}, o_{A}}^{A} E_{i_{B}, o_{B}}^{B}\right\}$, where the latter set includes measurement operators for every $i_{A}, o_{A}, i_{B}, o_{B}$. A more intuitive physical characterization of the almost-quantum set is also known [22]. A given $P_{O_{A}, O_{B} \mid I_{A}, I_{B}}$ belongs to the almost-quantum set if there exists a normalized quantum state $\rho$ and projective measurements $\left\{E_{i_{A}, o_{A}}^{A}\right\}$ and $\left\{E_{i_{B}, o_{B}}^{B}\right\}$ such that the correlations arise as usual via the Born rule:

$$
P_{O_{A}, O_{B} \mid I_{A}, I_{B}}\left(o_{A}, o_{B} \mid i_{A}, i_{B}\right)=\operatorname{Tr}\left[\rho\left(E_{i_{A}, o_{A}}^{A} E_{i_{B}, o_{B}}^{B}\right)\right] .
$$

However, crucially here we do not demand that the projectors corresponding to different parties commute. Instead we impose the following:

$$
E_{i_{A}, o_{A}}^{A} E_{i_{B}, o_{B}}^{B} \rho\left(E_{i_{A}, o_{A}}^{A} E_{i_{B}, o_{B}}^{B}\right)^{\dagger}=\Pi \rho \Pi^{\dagger},
$$

where $\Pi$ is any permutation of $E_{i_{A}, o_{A}}^{A} E_{i_{B}, o_{B}}^{B}$ (such as $\left.E_{i_{B}, o_{B}}^{B} E_{i_{A}, o_{A}}^{A}\right)$. Notice that this requirement does not ensure that 
the projectors for the two parties commute, so that the almostquantum set is an outer relaxation (a superset) of the quantum set. Interestingly, the almost-quantum set has been proven to satisfy many of the information-theoretic principles designed to pick out quantum theory from among all no-signaling theories, such as the local orthogonality principle, no advantage in nonlocal computation, etc. [14,23], also see [24,25] for interesting recent results in this direction. Moreover, a number of Bell inequalities achieve their optimal quantum violations already at this level, including the aforementioned correlation Bell inequalities.

\section{BELL INEQUALITIES WITH NO QUANTUM VIOLATION}

In identifying Bell inequalities for which no quantum violation exists, facet Bell inequalities play a crucial role. On the one hand, finding a facet Bell inequality with no quantum violation implies finding the largest dimensional face of the set of quantum correlations which one can describe analytically. On the other hand, if we relax the facet requirement, one can readily construct many Bell inequalities with no quantum violation by suitably tilting known facet Bell inequalities (that do admit quantum violation) [26].

(i) For instance, consider the well-studied $\mathrm{CHSH}$ Bell scenario $\mathbf{B}((2,2),(2,2))$, where Alice measures one of two binary observables $A_{1}, A_{2}$ and Bob similarly measures binary observables $B_{1}, B_{2}$. The classical polytope in this scenario is a well-characterized eight-dimensional polytope with the only nontrivial facet [the trivial facets are the non-negativity constraints $\left.P_{O_{A}, O_{B} \mid I_{A}, I_{B}}\left(o_{A}, o_{B} \mid i_{A}, i_{B}\right) \geqslant 0\right]$ known to be the $\mathrm{CHSH}$ inequality (up to local relabelings of inputs and outputs and an exchange of parties) given as

$$
\left\langle A_{1} B_{1}\right\rangle+\left\langle A_{1} B_{2}\right\rangle+\left\langle A_{2} B_{1}\right\rangle-\left\langle A_{2} B_{2}\right\rangle \leqslant 2,
$$

where as usual the correlator is $\left\langle A_{i_{A}} B_{i_{B}}\right\rangle=$ $\sum_{k=0,1}(-1)^{k} P_{O_{A}, O_{B} \mid i_{A}, i_{B}}\left(o_{A} \oplus o_{B}=k \mid i_{A}, i_{B}\right)$ for $i_{A}, i_{B}=1,2$. Tilting the above facet inequality by choosing coefficients $\alpha_{11}, \alpha_{12}, \alpha_{21}, \alpha_{22}>0$ normalized as $\alpha_{11}+\alpha_{12}+\alpha_{21}+\alpha_{22}=$ 1 , one gets the following class of inequalities:

$$
\begin{aligned}
& \alpha_{11}\left\langle A_{1} B_{1}\right\rangle+\alpha_{12}\left\langle A_{1} B_{2}\right\rangle+\alpha_{21}\left\langle A_{2} B_{1}\right\rangle-\alpha_{22}\left\langle A_{2} B_{2}\right\rangle \\
& \quad \leqslant 1-2 \min \left\{\alpha_{11}, \alpha_{12}, \alpha_{21}, \alpha_{22}\right\} .
\end{aligned}
$$

Using the Tsirelson solution for the quantum value of correlation Bell inequalities with binary outcomes, a simple characterization for the XOR games with no quantum advantage was obtained in [27]. We can use the characterization to show that (nonfacet) Bell inequalities of the form in (11) do not admit quantum violation when the following condition is satisfied by the coefficients (in the case when $\alpha_{22}<$ $\left.\alpha_{11}, \alpha_{12}, \alpha_{21}\right)$ [28]:

$$
\begin{aligned}
& \left(\alpha_{12} \alpha_{21}+\alpha_{11} \alpha_{22}\right)^{2} \\
& \quad \leqslant\left(\alpha_{11}+\alpha_{12}\right)\left(\alpha_{11}+\alpha_{21}\right)\left(\alpha_{12}-\alpha_{22}\right)\left(\alpha_{21}-\alpha_{22}\right) .
\end{aligned}
$$

An analogous condition holds when one of the other coefficients is the minimum as well. As an example satisfying the above condition, one may take $\left\{\alpha_{11}, \alpha_{12}, \alpha_{21}, \alpha_{22}\right\}=$ $\left\{\frac{9}{16}, \frac{1}{4}, \frac{1}{8}, \frac{1}{16}\right\}$. (ii) A second important consideration in finding Bell inequalities with no quantum violation is a recent breakthrough result [13] showing that any two-player XOR game, for which the corresponding Bell inequality is tight, has a quantum advantage. Their result, automatically rules out inequalities such as (11) under condition (12) and the XOR games with no quantum advantage derived in [23,27] from being facet Bell inequalities. However, binary-outcome correlation Bell inequalities only form a small subset of possible two-party Bell inequalities, since they restrict to the case $\delta_{A, i_{A}}=d_{B, i_{B}}=$ 2 for all $i_{A}, i_{B}$ and furthermore to the case that the inequality only consider terms involving the correlators $\left\langle A_{i_{A}} B_{i_{B}}\right\rangle$. Indeed, correlation Bell inequalities directly generalize to Bell scenarios where the number of outcomes for each party is $d>2$ leading to Bell inequalities of the type

$$
\begin{aligned}
& \sum_{i_{A}=1}^{m_{A}} \sum_{i_{B}=1}^{m_{B}} \sum_{o_{A}, o_{B}=1}^{d} q\left(i_{A}, i_{B}\right) \\
& \quad \times P_{O_{A}, O_{B} \mid i_{A}, i_{B}}\left(o_{A}+o_{B} \bmod d=f\left(i_{A}, i_{B}\right) \mid i_{A}, i_{B}\right) \leqslant \beta_{c},
\end{aligned}
$$

for some function $f$ from the inputs $\left(i_{A}, i_{B}\right)$ to a value in $\{1, \ldots, d\}$. Let the root of unity be $\zeta=\exp (2 \pi i / d)$, and define $d-1$ (game) matrices of dimension $m_{A} \times m_{B}$ as

$$
\Phi_{k}:=\sum_{i_{A}=1}^{m_{A}} \sum_{i_{B}=1}^{m_{B}} q\left(i_{A}, i_{B}\right) \zeta^{k f\left(i_{A}, i_{B}\right)}\left|i_{A}\right\rangle\left\langle i_{B}\right|,
$$

for $k=1, \ldots, d-1$. Then, a sufficient condition for Bell inequalities of the form (13) to have no quantum violation was shown by us in $[28,29]$. Namely, if the maximum left and right singular vectors $\left|u_{1}\right\rangle$ and $\left|v_{1}\right\rangle$ of $\Phi_{1}$ are composed entirely of roots of unity entries alone (arbitrary integral powers of $\zeta$ ), and simultaneously if the maximum left and right singular vectors $\left|u_{k}\right\rangle$ and $\left|v_{k}\right\rangle$ of $\Phi_{k}$ are obtainable from $\left|u_{1}\right\rangle$ and $\left|v_{1}\right\rangle$ by the substitution $\zeta \rightarrow \zeta^{k}$, then the corresponding inequality (13) admits no quantum violation. As an example consider the inequality corresponding to the game matrix

$$
\Phi_{1}=\frac{1}{24}\left[\begin{array}{cccc}
i & 2 & -2 & i \\
2 & i & i & -2 \\
-2 & i & i & 2 \\
i & -2 & 2 & i
\end{array}\right],
$$

i.e., with $f(1,1)=f(2,2)=f(3,3)=f(4,4)=f(1,4)=$ $f(2,3)=f(3,2)=f(4,1)=1, \quad f(1,2)=f(2,1)=$ $f(3,4)=f(4,3)=4, \quad f(1,3)=f(2,4)=f(3,1)=$ $f(4,2)=2$, and similarly $q(1,2)=q(2,1)=q(3,4)=$ $q(4,3)=q(1,3)=q(2,4)=q(3,1)=q(4,2)=\frac{1}{12} \quad$ and the remaining eight probabilities all equal to $\frac{1}{24}$. The corresponding inequality has classical value $\beta_{c}=\frac{3}{4}$ and an optimization to level $\mathbf{Q}_{1}[\mathbf{B}(2 ; 4,(4,4,4,4) ; 4,(4,4,4,4))]$ shows that the quantum value is also equal to $\beta_{q}=\frac{3}{4}$. This is reflected in the maximum singular vectors of $\Phi_{1}$ being composed of powers of $i=\exp (2 \pi i / 4)$ only, and the corresponding condition being satisfied by the matrices $\Phi_{2}, \Phi_{3}$ as well. The fact that the sufficient condition is inherited from the level $\mathbf{Q}_{1}\left[\mathbf{B}\left(2 ; m_{A}, \vec{d}_{A} ; m_{B}, \vec{d}_{B}\right)\right]$ in general [20] implies, by our central result, that none of the 
corresponding Bell inequalities with no quantum violation define facets of the Bell polytope.

(iii) Furthermore, the fact that XOR games obey a perfect parallel repetition theorem [30] implies that from a given binary-outcome correlation Bell inequality with no quantum violation, one can construct several Bell inequalities in higherdimensional multioutcome Bell scenarios that also do not allow for quantum violation. Indeed, any parallel repetition of the nonlocal computation game from [23] yields examples of $2^{k}$-output games without quantum advantage.

(iv) Even considering Bell inequalities with marginal terms, it is possible to construct inequalities with no quantum violation. We give an illustrative example here in the simple $\mathbf{B}((2,2),(2,2))$ scenario, more involved scenarios require a careful construction using the NPA hierarchy. Consider the inequality parametrized by real $0 \leqslant \alpha \leqslant 1$ and given as

$$
\begin{aligned}
P_{O_{A}, O_{B}} \mid i_{A}, i_{B} & (0,0 \mid 0,0)+\alpha P_{O_{A}, O_{B} \mid i_{A}, i_{B}}(1,1 \mid 0,0) \\
& \quad-P_{O_{A}, O_{B} \mid i_{A}, i_{B}}(0,1 \mid 0,1)-P_{O_{A}, O_{B} \mid i_{A}, i_{B}}(1,0 \mid 1,0) \\
& -P_{O_{A}, O_{B} \mid i_{A}, i_{B}}(0,0 \mid 1,1) \leqslant \alpha,
\end{aligned}
$$

where the classical maximum of $\alpha$ is readily obtained by direct inspection over local deterministic strategies. A wellknown result using Jordan's lemma [31] states that the quantum maximum of inequalities in this Bell scenario is ob- tainable by performing projective measurements on two-qubit states. A direct optimization over two-qubit states reveals that the inequality has the quantum value $\beta_{q}=\alpha$ in the parameter range $0.867 \leqslant \alpha<1$. Three affinely independent local deterministic strategies saturate the inequality, showing that the inequality defines a two-dimensional face of the set of local correlations. Together with the quantum strategy, at the critical value of $\alpha$ this saturates the bound of $m_{A}+\frac{1}{2} m_{A}\left(m_{A}-1\right)$ derived in [13]. The local strategies are explicitly given as follows: (i) Alice outputs $(1,1)$ for her two inputs $i_{A}=1,2$, Bob outputs $(1,0)$ for $i_{B}=1,2$, (ii) Alice outputs $(1,1)$, Bob outputs $(1,1)$, (iii) Alice outputs $(1,0)$, Bob outputs $(1,1)$.

\section{FACET BELL INEQUALITIES ARE VIOLATED IN ALMOST-QUANTUM THEORY}

Cabello, Severini, and Winter discovered a relationship between Bell scenarios (that also extends to more general contextuality scenarios) and graph theory [32,33]. For a given two-party Bell scenario $\mathbf{B}\left(\vec{d}_{A}, \vec{d}_{B}\right)$, one constructs an orthogonality graph $G_{\mathbf{B}\left(\vec{d}_{A}, \vec{d}_{B}\right)}$ as follows. Each input-output combination $\left(o_{A}, o_{B} \mid i_{A}, i_{B}\right)$ corresponds to a distinct vertex $v_{\left(o_{A}, o_{B} \mid i_{A}, i_{B}\right)}$ of the graph, and two such vertices are connected by an edge if the corresponding events are locally orthogonal, where local orthogonality is the condition that distinct outcomes are obtained for the same local input. In other words, we have

$$
v_{\left(o_{A}, o_{B} \mid i_{A}, i_{B}\right)} \sim v_{\left(o_{A}^{\prime}, o_{B}^{\prime} \mid i_{A}^{\prime}, i_{B}^{\prime}\right)} \Leftrightarrow\left(i_{A}=i_{A}^{\prime} \wedge o_{A} \neq o_{A}^{\prime}\right) \vee\left(i_{B}=i_{B}^{\prime} \wedge o_{B} \neq o_{B}^{\prime}\right) .
$$

Equivalently, we may consider that each product measurement operator $E_{i_{A}, o_{A}}^{A} E_{i_{B}, o_{B}}^{B}$ corresponds to a vertex in the graph $G_{\mathbf{B}}\left(\vec{d}_{A}, \vec{d}_{B}\right)$ with vertices connected by an edge if $i_{A}=i_{A}^{\prime}$ and $E_{i_{A}, o_{A}}^{A} E_{i_{A}^{\prime}, o_{A}^{\prime}}^{A}=0$ or $i_{B}=i_{B}^{\prime}$ and $E_{i_{B}, o_{B}}^{B} E_{i_{B}^{\prime}, o_{B}^{\prime}}^{B}=0$. The number of vertices in the graph is $\left|V\left(G_{\mathbf{B}\left(\vec{d}_{A}, \vec{d}_{B}\right)}\right)\right|=n$.

Furthermore, given a graph $G$ with vertex set $V(G)$ and edge set $E(G)$ one can also find a set of unit vectors obeying the above orthogonality conditions, called an orthonormal representation of the graph. Formally, an orthonormal representation of graph $G$ is a set $\left\{\left|u_{i}\right\rangle \in \mathbb{R}^{N}: i \in V(G)\right\}$ where $N$ is some arbitrary dimension, $\|\left|u_{i}\right\rangle \|=1$ for all $i \in V(G)$ and $\left\langle u_{i} \mid u_{j}\right\rangle=0$ for $(i, j) \in E(G)$. It should be noted that in the graph-theoretic literature, the Lovász orthogonal representation is also defined in a complementary manner, with nonadjacent vertices being assigned orthogonal vectors. For a given graph $G$, the Lovász theta-body $\mathrm{TH}(G)$ (sometimes also called the Grötschel-Lovász-Schrijver theta-body) is a convex set introduced [34-36] as a semidefinite programming relaxation to the hard graph-theoretic problem of finding a maximum weight stable set of the graph (a stable set is a set of mutually nonadjacent vertices). The theta set is defined as follows:

Definition 1. For a graph $G=(V(G), E(G))$, define the convex set $T H(G)$ as

$$
\operatorname{TH}(G):=\left\{|\mathcal{P}\rangle=\left(\left|\left\langle\psi \mid u_{i}\right\rangle\right|^{2}: i \in V(G)\right) \in \mathbb{R}_{+}^{V(G)}|\|| \psi\right\rangle\|=\|\left|u_{i}\right\rangle \|=1,
$$

The similarity between the set $\operatorname{TH}(G)$ and the set $\mathbf{Q}_{1+A B}\left[\mathbf{B}\left(2 ; m_{A}, \vec{d}_{A} ; m_{B}, \vec{d}_{B}\right)\right]$ has been noted in the literature, here we give a self-contained proof that is more suited towards establishing our main result. First, as shown in [14], the normalization and no-signaling constraints on a box can be rewritten in terms of maximum clique equalities in the orthogonality graph, where a clique inequality is an inequality of the form

$$
\sum_{v_{\left(o_{A}, o_{B} \mid i_{A}, i_{B}\right)} \in c} P_{O_{A}, O_{B} \mid I_{A}, I_{B}}\left(o_{A}, o_{B} \mid i_{A}, i_{B}\right) \leqslant 1,
$$


for some clique $c$ in the graph. Here a clique denotes a set of mutually adjacent vertices. Now, since by definition, each normalization constraint only considers events corresponding to different outcomes for the same measurement setting, it is clear that the normalization constraint corresponds to a clique inequality that is saturated. To see that the no-signaling condition also corresponds to such a constraint, note that using the normalization constraint, the no-signaling conditions can be rewritten in the form

$$
\begin{gathered}
\sum_{o_{A}=1}^{d_{A, i_{A}}} P_{O_{A}, O_{B} \mid I_{A}, I_{B}}\left(o_{A}, o_{B} \mid i_{A}, i_{B}\right)+\sum_{\substack{o_{B}^{\prime}=1 \\
o_{B}^{\prime} \neq o_{B}}}^{d_{B, i_{B}}^{\prime}} \sum_{o_{A}^{\prime}=1}^{d_{A, i_{A}^{\prime}}} P_{O_{A}, O_{B} \mid I_{A}, I_{B}}\left(o_{A}^{\prime}, o_{B}^{\prime} \mid i_{A}^{\prime}, i_{B}\right)=1 \text { for all } i_{A}, i_{A}^{\prime}, o_{B}, i_{B}, \\
\sum_{o_{B}=1}^{d_{B, i_{B}}} P_{O_{A}, O_{B} \mid I_{A}, I_{B}}\left(o_{A}, o_{B} \mid i_{A}, i_{B}\right)+\sum_{\substack{o_{A}^{\prime}=1 \\
o_{A}^{\prime} \neq o_{A}}}^{d_{A, i_{A}}} \sum_{o_{B}^{\prime}=1}^{d_{B, i_{B}^{\prime}}} P_{O_{A}, O_{B} \mid I_{A}, I_{B}}\left(o_{A}^{\prime}, o_{B}^{\prime} \mid i_{A}, i_{B}^{\prime}\right)=1 \text { for all } i_{B}, i_{B}^{\prime}, o_{A}, i_{A} .
\end{gathered}
$$

Each no-signaling condition expressed in the above form considers events that are locally orthogonal, and thus corresponds to a saturated clique inequality. Furthermore, the normalization and no-signaling conditions correspond to maximum clique inequalities, i.e., no other measurement event $\left(o_{A}, o_{B} \mid i_{A}, i_{B}\right)$ exists that is locally orthogonal to every event in these equations. Interestingly, it was shown in [14] that in any two-party Bell scenario $\mathbf{B}\left(\vec{d}_{A}, \vec{d}_{B}\right)$, the normalization and no-signaling conditions encompass all the maximum clique inequalities, i.e., every maximum clique inequality corresponds to a normalization or a no-signaling constraint. On the other hand, when one considers Bell scenarios involving three or more parties, other maximum clique inequalities exist, and these are the constraints identified by the local orthogonality principle.

In a formal sense, $\mathbf{Q}_{1+A B}\left[\mathbf{B}\left(2 ; m_{A}, \vec{d}_{A} ; m_{B}, \vec{d}_{B}\right)\right]$ is equivalent to the set $\operatorname{TH}(G)$ defined for an appropriate orthogonality graph $G_{\mathbf{B}\left(\vec{d}_{A}, \vec{d}_{B}\right)}$, with the additional constraint that the maximum clique inequalities corresponding to the normalization and the no-signaling conditions be set to equalities. In other words, define $\mathcal{C}_{n, n s}$ as the set of maximum cliques in the orthgonality graph $G_{\mathbf{B}\left(\vec{d}_{A}, \vec{d}_{B}\right)}$ that correspond to the normalization and no-signaling constraints in the Bell scenario. Define the convex set $\mathrm{TH}_{n, n s}\left(G_{\mathbf{B}\left(\vec{d}_{A}, \vec{d}_{B}\right)}\right)$ as

$$
\mathrm{TH}_{n, n s}\left(G_{\mathbf{B}\left(\vec{d}_{A}, \vec{d}_{B}\right)}\right):=\left\{|\mathcal{P}\rangle=\left[\left|\left\langle\psi \mid u_{i}\right\rangle\right|^{2}: i \in V\left(G_{\mathbf{B}\left(\vec{d}_{A}, \vec{d}_{B}\right)}\right)\right] \in \mathbb{R}_{+}^{n} \mid \begin{array}{l}
\||\psi\rangle\|=\|\left|u_{i}\right\rangle \|=1 \forall i \\
\left\{\left|u_{i}\right\rangle\right\} \text { is an orth. repn. of } G_{\mathbf{B}\left(\vec{d}_{A}, \vec{d}_{B}\right)} \\
\sum_{i \in c}\left|\left\langle\psi \mid u_{i}\right\rangle\right|^{2}=1, \quad \text { for all } c \in \mathcal{C}_{n, n s}
\end{array}\right\} .
$$

The set $\mathbf{Q}_{1+A B}\left[\mathbf{B}\left(2 ; m_{A}, \vec{d}_{A} ; m_{B}, \vec{d}_{B}\right)\right]$ is then equivalent to $\mathrm{TH}_{n, n s}\left(G_{\mathbf{B}\left(\vec{d}_{A}, \vec{d}_{B}\right)}\right)$ :

Theorem 2 (see [32,37,38]). For any two-party Bell scenario $\mathbf{B}\left(\vec{d}_{A}, \vec{d}_{B}\right)$, it holds that $\mathbf{Q}_{1+A B}\left[\mathbf{B}\left(\vec{d}_{A}, \vec{d}_{B}\right)\right]=$ $\mathrm{TH}_{n, n s}\left(G_{\mathbf{B}\left(\vec{d}_{A}, \vec{d}_{B}\right)}\right)$.

At this point it is important to note the dimension mismatch between the sets $\mathbf{Q}_{1+A B}\left[\mathbf{B}\left(2 ; m_{A}, \vec{d}_{A} ; m_{B}, \vec{d}_{B}\right)\right]$ and $\mathrm{TH}(G)$. Namely, while $\mathrm{TH}(G)$ is a full-dimensional convex set (of dimension $n$ ), $\mathbf{Q}_{1+A B}\left[\mathbf{B}\left(2 ; m_{A}, \vec{d}_{A} ; m_{B}, \vec{d}_{B}\right)\right]$ is of much smaller dimension (being of dimension $D$ ). Therefore, one may wonder whether any statements about the facets of $\operatorname{TH}(G)$ hold true for the smaller dimensional set, since facets of $\mathbf{Q}_{1+A B}\left[\mathbf{B}\left(2 ; m_{A}, \vec{d}_{A} ; m_{B}, \vec{d}_{B}\right)\right]$ would be faces of much smaller dimension in $\mathrm{TH}(G)$. Nevertheless, we use techniques used in the study of the facets of $\operatorname{TH}(G)$ to show the following statement about the facets of the almost-quantum set.
Theorem 3. In bipartite Bell scenarios, irrespective of the number of inputs and outputs for each party, the only fulldimensional linear facets of the almost-quantum set are also facets of the no-signaling polytope. In other words, for a facet Bell inequality of the form

$$
\sum_{o_{A}, o_{B}, i_{A}, i_{B}} q\left(i_{A}, i_{B}\right) V\left(o_{A}, o_{B}, i_{A}, i_{B}\right) P\left(o_{A}, o_{B} \mid i_{A}, i_{B}\right) \leqslant \omega_{c},
$$

where $\omega_{c}$ denotes the classical value of the inequality, the almost-quantum value $\omega_{\tilde{q}}$ is strictly larger than $\omega_{c}$, i.e., $\omega_{\tilde{q}}>$ $\omega_{c}$.

Proof. The proof follows analogously to that of an analogous claim made for the general Lovász theta set $\mathrm{TH}(G)$. It is noteworthy that the set $\mathrm{TH}(G)$ has been characterized in multiple ways in the literature. We begin with a complementary characterization of the set $\mathrm{TH}_{n, n s}\left(G_{\mathbf{B}\left(\vec{d}_{A}, \vec{d}_{B}\right)}\right)$ inherited from a characterization of $\mathrm{TH}(G)[39,40]$ that is particularly suited to our problem:




Here $\bar{G}$ denotes the graph complement of $G$, i.e., the graph with the same vertex set as $G$ and the complementary edge set $(u \sim v$ in $\bar{G} \Leftrightarrow u \nsim v$ in $G)$. This representation of $\mathrm{TH}_{n, n s}\left(G_{\mathbf{B}\left(\vec{d}_{A}, \vec{d}_{B}\right)}\right)=\mathbf{Q}_{1+A B}\left[\mathbf{B}\left(2 ; m_{A}, \vec{d}_{A} ; m_{B}, \vec{d}_{B}\right)\right]$ is useful since it characterizes the facets of the set, in particular every facet is of the form $\sum_{i \in V\left(G_{\mathbf{B}\left(\vec{d}_{A}, \vec{d}_{B}\right)}\right)}\left|\left\langle\phi \mid w_{i}\right\rangle\right|^{2}|\mathcal{P}\rangle_{i}=1$ for some unit vector $|\phi\rangle \in \mathbb{R}^{N}$ and orthonormal representation $\left\{\left|w_{i}\right\rangle \in \mathbb{R}^{N}\right\}$ of $\bar{G}$. It is also worth noting that the normalization and no-signaling constraints $\sum_{i \in c}|\mathcal{P}\rangle_{i}=1$ also fall in this category, if we choose $\left|w_{i}\right\rangle=|\phi\rangle$ for every vertex $i \in c$ and $\left|w_{i}\right\rangle=|\phi\rangle^{\perp}$, for some arbitrary unit vector $|\phi\rangle$ and an orthogonal unit vector $|\phi\rangle^{\perp} \perp|\phi\rangle$.

Let $F=\left\{\left.|P\rangle\left|\sum_{i \in V\left(G_{\mathbf{B}\left(\vec{d}_{A}, \vec{d}_{B}\right)}\right.}\right|\left\langle\phi \mid w_{i}\right\rangle\right|^{2}|\mathcal{P}\rangle_{i}=1\right\}$ be a facet of $\mathbf{Q}_{1+A B}\left[\mathbf{B}\left(2 ; m_{A}, \vec{d}_{A} ; m_{B}, \vec{d}_{B}\right)\right]$. Let $\left|\mathcal{P}^{*}\right\rangle \in \operatorname{int}(F)$. We have the following:

$$
\begin{gathered}
\sum_{i \in V\left(G_{\mathbf{B}\left(\vec{d}_{A}, \vec{d}_{B}\right)}\right)}\left|\left\langle\phi \mid w_{i}\right\rangle\right|^{2}\left|\mathcal{P}^{*}\right\rangle_{i} \leqslant\langle\phi \mid \phi\rangle \\
\Rightarrow\left\langle\phi\left|\left(\sum_{i \in V\left(G_{\mathbf{B}\left(\vec{d}_{A}, \vec{d}_{B}\right)}\right)}\left|\mathcal{P}^{*}\right\rangle_{i}\left|w_{i}\right\rangle\left\langle w_{i}\right|\right)\right| \phi\right\rangle \leqslant 1 .
\end{gathered}
$$

Saturation of the above inequality implies that $|\phi\rangle$ is a maximum eigenvector of $\left(\sum_{i \in V\left(G_{\mathbf{B}\left(\vec{d}_{A}, \vec{d}_{B}\right)}\right)}\left|\mathcal{P}^{*}\right\rangle_{i}\left|w_{i}\right\rangle\left\langle w_{i}\right|\right)$ corresponding to eigenvalue 1 . In other words

$$
\begin{aligned}
& \left(\sum_{i \in V\left(G_{\mathbf{B}\left(\vec{d}_{A}, \vec{d}_{B}\right)}\left|\mathcal{P}^{*}\right\rangle_{i}\left|w_{i}\right\rangle\left\langle w_{i}\right|\right)|\phi\rangle=|\phi\rangle,}\right. \\
& \quad \times\left(\sum_{i \in V\left(G_{\mathbf{B}\left(\vec{d}_{A}, \vec{d}_{B}\right)}\right)}\left|\mathcal{P}^{*}\right\rangle_{i}\left\langle w_{i} \mid \phi\right\rangle\right)\left|w_{i}\right\rangle_{j} \\
& =\left(\sum_{i \in V\left(G_{\mathbf{B}\left(\vec{d}_{A}, \vec{d}_{B}\right)}\right)}\left|\mathcal{P}^{*}\right\rangle_{i}\left|\left\langle w_{i} \mid \phi\right\rangle\right|^{2}\right)|\phi\rangle_{j} \text { for } j=1, \ldots, N,
\end{aligned}
$$

where in obtaining the last equation we have used the first inequality of (23). Now as $F$ is a facet, it is not a convex combination of other facet-defining inequalities in Eq. (22), so that the above equality implies

$$
\left\langle w_{i} \mid \phi\right\rangle\left|w_{i}\right\rangle=\left|\left\langle w_{i} \mid \phi\right\rangle\right|^{2}|\phi\rangle \quad \forall i \in V\left(G_{\mathbf{B}\left(\vec{d}_{A}, \vec{d}_{B}\right)}\right) .
$$

This gives that for each $i \in V\left(G_{\mathbf{B}\left(\vec{d}_{A}, \vec{d}_{B}\right)}\right)$, either $\left\langle w_{i} \mid \phi\right\rangle=0$ or

$$
\begin{aligned}
\left|w_{i}\right\rangle & =\left\langle w_{i} \mid \phi\right\rangle|\phi\rangle \\
\Rightarrow\left|w_{i}\right\rangle & = \pm|\phi\rangle,
\end{aligned}
$$

without loss of generality we may take $\left|w_{i}\right\rangle=|\phi\rangle$. Therefore, for every $i \in V\left(G_{\mathbf{B}\left(\vec{d}_{A}, \vec{d}_{B}\right)}\right)$, either $\left\langle w_{i} \mid \phi\right\rangle=0$ or $\left|w_{i}\right\rangle=|\phi\rangle$.

Defining the set $\mathcal{I}$ as $\left.\mathcal{I}:=\left\{i \in V\left(G_{\mathbf{B}\left(\vec{d}_{A}, \vec{d}_{B}\right)}\right)|| w_{i}\right\rangle=|\phi\rangle\right\}$, we obtain that $\left\{\left|w_{i}\right\rangle\right\}$ is an orthonormal representation of $\bar{G}$ where $\left|w_{i}\right\rangle$ takes value $|\phi\rangle$ for every $i \in \mathcal{I}$, while $\left|w_{i}\right\rangle$ belongs to the subspace of $\mathbb{R}^{N}$ that is orthogonal to $|\phi\rangle$ when $i \notin \mathcal{I}$. This therefore implies that all the vertices in $\mathcal{I}$ are mutually nonadjacent in $\bar{G}$, i.e., that $\mathcal{I}$ is a stable set of $\bar{G}$ or in other words $\mathcal{I}$ is a clique of $G$. The inequality $\sum_{i \in V\left(G_{\mathbf{B}\left(\vec{d}_{A}, \vec{d}_{B}\right)}\right)}\left|\left\langle\phi \mid w_{i}\right\rangle\right|^{2}|\mathcal{P}\rangle_{i}=1$ supporting facet $F$ of $\mathbf{Q}_{1+A B}\left[\mathbf{B}\left(2 ; m_{A}, \vec{d}_{A} ; m_{B}, \vec{d}_{B}\right)\right]$ thus constitutes the saturation of a clique inequality of the form $\sum_{i \in \mathcal{I}}|\mathcal{P}\rangle_{i}=1$. Now from [14,41] we know that in any twoparty Bell scenario, the clique inequalities are exhausted by the no-signaling and normalization constraints. Therefore, no other proper facet-defining Bell inequality exists that is also a facet of $\mathbf{Q}_{1+A B}\left[\mathbf{B}\left(2 ; m_{A}, \vec{d}_{A} ; m_{B}, \vec{d}_{B}\right)\right]$, i.e., every twoparty facet Bell inequality is violated in almost-quantum theory.

Thm. (3) thus shows that every two-party facet Bell inequality, irrespective of the number of inputs and outputs for each party, admits a violation in almost-quantum theory. It is worth noting that an alternative route to deriving the result in Thm. (3) is to parametrize box $|\mathcal{P}\rangle$ in terms of $D$ parameters, being probabilities $P_{O_{A}, O_{B} \mid I_{A}, I_{B}}\left(o_{A}, o_{B} \mid i_{A}, i_{B}\right)$, from which other probabilities that define the box can be obtained via normalization and no-signaling conditions, as done for example in [21]. The result on facets of $\operatorname{TH}(G)$ from [39,40,42] applied to the theta-set of the orthogonality graph of this subset of events in the Bell experiment, can then be used to derive Thm. (3).

Corollary 4. Any two-party facet Bell inequality, irrespective of the number of inputs and outputs for each party, for which the quantum value is achieved at level $1+A B$ of the NPA hierarchy, admits a violation in quantum theory. In particular, two-party XOR games that define facet Bell inequalities always admit a quantum advantage.

Proof. Two-party XOR games form a class of Bell inequalities for which the quantum value is achieved at level $1+A B$, in fact already at level 1 of the NPA hierarchy, by the results of Tsirelson [20]. Therefore, binary-outcome correlation Bell inequalities that do not admit quantum violation, do not define facets of the classical Bell polytope.

This Corollary neatly recovers the result by Escolá et al. [13]. Besides, as we have seen it also recovers a central result of [28], namely that $d$-outcome nonlocal computation games do not define facets of the Bell polytope. Finally, other unique games with no quantum advantage considered in [28] are also shown to not correspond to facet-defining Bell inequalities. An important question remains.

Open Can the method be extended to other levels of the NPA hierarchy, to identify whether any two-party facet Bell inequality also defines a facet of the set of quantum correlations?

Such a facet, if it exists, could provide the crucial insight towards identifying a fundamental information-theoretic principle that explains why nature chose quantum theory over almost-quantum theory [22]. The result shown here does not indicate the answer to the question in either direction, since it is at present unknown how rare are the linear inequalities which admit almost-quantum violation but no quantum violation. 


\section{ALL TWO-PARTY FACET-DEFINING INEQUALITIES OF THE BELL CORRELATION POLYTOPE ARE VIOLATED IN QUANTUM THEORY}

Avis et al. [12] posed the question whether there are any facets of the binary-outcome correlation polytope (the classical polytope of two-party binary-outcome correlations $\left\langle A_{i_{A}} B_{i_{B}}\right\rangle \in\{+1,-1\}$, excluding the local marginal terms) that are not violated in quantum theory. In other words, is there any nontrivial facet of the set of classical bipartite two-point correlation vectors (expectation values of the form $\left\langle A_{i_{A}} B_{i_{B}}\right\rangle \in$ $\{+1,-1\})$ that is not violated in quantum theory? Note that these sets are of smaller dimension than the sets considered in the previous sections, since they only involve the two-point correlation terms and not the local marginal terms $\left\langle A_{i_{A}}\right\rangle$ and $\left\langle B_{i_{B}}\right\rangle$.

The question raised by Avis et al. [12] was recently answered in the negative by Escolá et al. [13], making use of the simple characterization of XOR games with no quantum advantage given in [27]. Here we provide an alternative proof, making use of a connection between the correlation polytope and the cut polytope of graph theory [12]. In particular, this connection links the set of binary-outcome quantum correlations and the well-studied elliptope in graph theory, this latter body being the semidefinite programming relaxation of the cut polytope.

First, we introduce the cut polytope of complete graph following [43]. The graph is denoted by $\Gamma_{t}$, has $t$ vertices, and has an edge between each pair of vertices. A cut $S$ is an assignment of $\{0,1\}$ to each vertex in the graph. The cut vector $\delta(S)$ for some cut $S$ is given by $\delta_{u, v}(S)=1$ if vertices $u, v$ are assigned different values, and 0 if the vertices are assigned the same values. The set of all convex combinations of cut vectors $\operatorname{Cut}\left(\Gamma_{t}\right)=\left\{\sum_{S: \text { cut }} p_{S} \delta(S) \mid \sum_{S: \text { cut }} p_{S}=1, p_{S} \geqslant 0\right\}$ is called the cut polytope of the complete graph. The vectors of correlation functions which are possible in classical correlation experiments form the cut polytope $\operatorname{Cut}\left(\Gamma_{m_{A}, m_{B}}\right)$ of the complete bipartite graph $\Gamma_{m_{A}, m_{B}}$. Tight correlation inequalities are exactly the facet-inducing inequalities of the polytope $\operatorname{Cut}\left(\Gamma_{m_{A}, m_{B}}\right)$.

The semidefinite relaxation of the cut polytope of the complete graph $\Gamma_{t}$ is the elliptope $\mathcal{E}\left(\Gamma_{t}\right)$ also sometimes denoted as $\mathcal{E}_{t \times t}$. Formally, $\mathcal{E}_{t \times t}$ denotes the set of $t \times t$ correlation matrices (positive semidefinite matrices with diagonal entries equal to 1 )

$$
\mathcal{E}_{t \times t}:=\left\{M \in \mathbb{R}^{t \times t} \mid M \succeq 0, M_{i, i}=1 \text { for all } i=1, \ldots, t\right\} .
$$

In general, the elliptope $\mathcal{E}(G)$ of a graph $G=(V, E)$ with $|V|$ vertices is the convex body consisting of vectors $\vec{x} \in \mathbb{R}^{E}$ such that there exist a unit vector $\left|u_{i}\right\rangle \in \mathbb{R}^{|V|}$ for each vertex $i \in V$ satisfying $\vec{x}_{i, j}=\left\langle u_{i} \mid u_{j}\right\rangle$. In particular, the elliptope of the complete bipartite graph $\mathcal{E}\left(\Gamma_{m_{A}, m_{B}}\right)$ is the set of vectors $\vec{x} \in \mathbb{R}^{E\left(\Gamma_{m_{A}, m_{B}}\right)}$ satisfying the conditions of Tsirelson's theorem, so that $\mathcal{E}\left(\Gamma_{m_{A}, m_{B}}\right)$ is the set of bipartite binary-outcome quantum correlations [12]. The dimensionalities of these sets is $\operatorname{dim}\left(\mathcal{E}_{t \times t}\right)=\left(\begin{array}{l}t \\ 2\end{array}\right)$, and $\operatorname{dim}\left[\mathcal{E}\left(\Gamma_{m_{A}, m_{B}}\right)\right]=m_{A} m_{B}$. Moreover, $\mathcal{E}\left(\Gamma_{m_{A}, m_{B}}\right)$ is a projection of $\mathcal{E}_{t \times t}$ onto the lower-dimensional space for $t=m_{A}+m_{B}$.
We now show that every two-party facet-defining correlation Bell inequality, irrespective of the number of inputs and outputs for each party, admits a violation in quantum theory. In other words, for a facet-defining correlation Bell inequality of the form

$$
\sum_{i_{A}, i_{B}} \alpha_{i_{A}, i_{B}}\left\langle A_{i_{A}} B_{i_{B}}\right\rangle \leqslant \beta_{c},
$$

where $\beta_{c}$ denotes the classical value of the inequality, the quantum value $\beta_{q}$ is strictly larger than $\beta_{c}$. Formally, we show the following.

Theorem 5. All faces of the set of quantum bipartite twopoint correlation vectors are of strictly lower dimension than that of the facets of the set of classical bipartite two-point correlation vectors.

The proof of Thm. (5) follows from the discussion above, mapping the cut polytope and the correlation Bell polytope, along with the corresponding mapping between the elliptope and the set of two-party binary-outcome quantum correlations [12]. Laurent and Poljak in [44], building upon the results of [45], show that the largest dimension of a polyhedral face (formed by the convex hull of cut vectors) of $\mathcal{E}_{t \times t}$ is equal to the largest integer $d_{t}$ such that $\left(\begin{array}{c}d_{t}+1 \\ 2\end{array}\right) \leqslant t-1$, i.e., $d_{t}=$ $\left\lfloor\frac{\sqrt{8 t-7}-1}{2}\right\rfloor$. They further show that the largest dimension of any face of $\mathcal{E}_{t \times t}$ is $\left(\begin{array}{c}t-1 \\ 2\end{array}\right)$. A facet-defining correlation Bell inequality is, by definition, of dimension $\operatorname{dim}\left[\mathcal{E}\left(\Gamma_{m_{A}, m_{B}}\right]\right)-1=$ $m_{A} m_{B}-1$. For $t:=m_{A}+m_{B}$, it is readily seen that for all values of $m_{A}, m_{B} \geqslant 2$,

$$
m_{A} m_{B}-1>d_{t},
$$

so that the set of quantum bipartite two-point correlation vectors shares no facets with the set of classical bipartite two-point correlation vectors. It follows that all bipartite Bell inequalities which are facets of the classical correlation polytope must admit quantum violation.

\section{CONCLUSIONS}

In this paper we have shown that that all two-party Bell inequalities that define facets of the classical Bell polytope, are violated in a natural semidefinite programming relaxation to the set of quantum correlations, termed almost-quantum theory. We have also seen that all correlation Bell inequalities that define facets of the lower dimensional correlation Bell polytope, are violated in quantum theory.

We reiterate the important open question that still remains.

Open Is every facet-defining bipartite Bell inequality (of the classical two-party Bell polytope) violated in quantum theory?

A positive answer for the question could provide a promising route to identifying a fundamental principle singling out the set of quantum correlations among other nonsignaling ones. It would be interesting to see if the methods discussed here can be extended to further levels of the convergent hierarchy of semidefinite programming relaxations of the quantum set. It would also be interesting to find tight bounds on the dimension of the faces of the quantum set, and informationtheoretic explanations behind these. 


\section{ACKNOWLEDGMENTS}

We acknowledge useful discussions with Stefano Pironio, Paweł Horodecki, Jed Kaniewski, and Andreas Winter. We also thank an anonymous referee for their valuable comments. This work is supported by the Start-up Fund "Device-Independent Quantum Communication Networks" from The University of Hong Kong (166DRRAVI), the Seed Fund "Security of Relativistic Quantum Cryptography" (Grant No. 201909185030) and the Early Career Scheme (ECS) grant "Device-Independent Random Number Gener- ation and Quantum Key Distribution with Weak Random Seeds" (Grant No. 27210620). This work was supported by the National Natural Science Foundation of China through Grant No. 11675136, the Hong Kong Research Grant Council through Grant No. 17300918, and the John Templeton Foundation through Grants 60609, Quantum Causal Structures, and 61466, The Quantum Information Structure of Spacetime (qiss.fr). The opinions expressed in this publication are those of the author and do not necessarily reflect the views of the John Templeton Foundation.
[1] A. K. Ekert, Quantum Cryptography Based on Bell's Theorem, Phys. Rev. Lett. 67, 661 (1991).

[2] A. Acín, N. Brunner, N. Gisin, S. Massar, S. Pironio, and V. Scarani, Device-Independent Security of Quantum Cryptography against Collective Attacks, Phys. Rev. Lett. 98, 230501 (2007).

[3] S. Pironio, A. Acín, S. Massar, A. B. de la Giroday, D. N. Matsukevich, P. Maunz, S. Olmschenk, D. Hayes, L. Luo, T. A. Manning, and C. Monroe, Random numbers certified by Bell's theorem, Nature (London) 464, 1021 (2010).

[4] R. Colbeck, Quantum and relativistic protocols for secure multiparty computation, Ph.D. thesis, University of Cambridge, 2007.

[5] R. Colbeck and R. Renner, Free randomness can be amplified, Nat. Phys. 8, 450 (2010).

[6] F. G. S. L. Brandão, R. Ramanathan, A. Grudka, K. Horodecki, M. Horodecki, P. Horodecki, T. Szarek, and H. Wojewódka, Realistic noise-tolerant randomness amplification using finite number of devices, Nat. Commun. 7, 11345 (2016).

[7] H. Buhrman, R. Cleve, S. Massar, and R. de Wolf, Nonlocality and communication complexity, Rev. Mod. Phys. 82, 665 (2010).

[8] K. T. Goh, J. Kaniewski, E. Wolfe, T. Vértesi, X. Wu, Y. Cai, Y.-C. Liang, and V. Scarani, Geometry of the set of quantum correlations, Phys. Rev. A 97, 022104 (2018).

[9] I. Pitowsky, Quantum Probability - Quantum Logic, Lecture Notes in Physics, Vol. 321 (Springer, Berlin, 1989).

[10] C. Śliwa, Symmetries of the Bell correlation inequalities, Phys. Lett. A 317, 165 (2003).

[11] R. Gill, Some open problems in quantum information theory, arXiv:quant-ph/0504166.

[12] D. Avis, H. Imai, and T. Ito, On the relationship between convex bodies related to correlation experiments with dichotomic observables, J. Phys. A: Math. Gen. 39, 36 (2006).

[13] L. Escolá, J. Calsamiglia, and A. Winter, All tight correlation Bell inequalities have quantum violations, Phys. Rev. Res. 2, 012044(R) (2020).

[14] T. Fritz, A. B. Sainz, R. Augusiak, J. B. Brask, R. Chaves, A. Leverrier, and A. Acín, Local orthogonality as a multipartite principle for quantum correlations, Nat. Commun. 4, 2263 (2013).

[15] M. L. Almeida, J.-D. Bancal, N. Brunner, A. Acín, N. Gisin, and S. Pironio, Guess Your Neighbor's Input: A Multipartite Nonlocal Game with No Quantum Advantage, Phys. Rev. Lett. 104, 230404 (2010).
[16] R. Augusiak, T. Fritz, Ma. Kotowski, Mi. Kotowski, M. Pawłowski, M. Lewenstein, and A. Acín, Tight Bell inequalities with no quantum violation from qubit unextendible product bases, Phys. Rev. A 85, 042113 (2012).

[17] J. F. Clauser, M. A. Horne, A. Shimony, and R. A. Holt, Proposed Experiment to Test Local Hidden-Variable Theories, Phys. Rev. Lett. 23, 880 (1969).

[18] S. Pironio, Lifting Bell inequalities, J. Math. Phys. 46, 062112 (2005).

[19] D. Avis, H. Imai, T. Ito, and Y. Sasaki, Two-party Bell inequalities derived from combinatorics via triangular elimination, J. Phys. A: Math. Gen. 38, 10971 (2005).

[20] B. S. Cirel'son, Quantum generalizations of Bell's inequality, Lett. Math. Phys. 4, 93 (1980).

[21] M. Navascués, S. Pironio, and T. Acín, A convergent hierarchy of semidefinite programs characterizing the set of quantum correlations, New J. Phys. 10, 073013 (2008).

[22] M. Navascués, Y. Guryanova, M. J. Hoban, and A. Acín, Almost quantum correlations, Nat. Commun. 6, 6288 (2015).

[23] N. Linden, S. Popescu, A. J. Short, and A. Winter, Quantum Nonlocality and Beyond: Limits from Nonlocal Computation, Phys. Rev. Lett. 99, 180502 (2007).

[24] T. Gonda, R. Kunjwal, D. Schmid, E. Wolfe, and A. B. Sainz, Almost quantum correlations are inconsistent with Specker's principle, Quantum 2, 87 (2018).

[25] A. B. Sainz, Y. Guryanova, A. Acín, and M. Navascues, AlmostQuantum Correlations Violate the No-Restriction Hypothesis, Phys. Rev. Lett. 120, 200402 (2018).

[26] D. Goyeneche, W. Bruzda, O. Turek, D. Alsina, and K. Życzkowski, The powerfulness of classical correlations, arXiv:2004.00695.

[27] R. Ramanathan, A. Kay, G. Murta, and P. Horodecki, Characterizing the Performance of XOR Games and the Shannon Capacity of Graphs, Phys. Rev. Lett. 113, 240401 (2014).

[28] R. Ramanathan, M. T. Quintino, A. B. Sainz, G. Murta, R Augusiak, On the tightness of correlation inequalities with no quantum violation, Phys. Rev. A 95, 012139 (2017).

[29] R. Ramanathan, R. Augusiak, and G. Murta, Generalized XOR games with $d$ outcomes and the task of nonlocal computation, Phys. Rev. A 93, 022333 (2016).

[30] R. Cleve, W. Slofstra, F. Unger, and S. Upadhyay, Perfect parallel repetition theorem for quantum XOR proof systems, in Twenty-Second Annual IEEE Conference on Computational Complexity (CCC'07) (IEEE, Piscataway, NJ, 2007).

[31] C. Jordan, Essai sur la geometrie a n dimensions, Bull. S. M. F. 3, 103 (1875). 
[32] A. Cabello, S. Severini, and A. Winter, (Non-)contextuality of physical theories as an axiom, arXiv:1010.2163.

[33] A. Cabello, S. Severini, and A. Winter, Graph-Theoretic Approach to Quantum Correlations, Phys. Rev. Lett. 112, 040401 (2014).

[34] M. Grötschel, L. Lovász, and A. Schrijver, Relaxations of vertex packing, J. Combin. Theory Ser. B 40, 330 (1986).

[35] M. Grötschel, L. Lovász, and A. Schrijver, in Topics on Perfect Graphs, North-Holland Mathematical Studies, Vol. 88 (NorthHolland, Amsterdam, 1984), p. 325.

[36] A. Schrijver, Combinatorial Optimization. Polyhedra and Efficiency, Vol. A, Series volume 24 of Algorithms and Combinatorics (Springer, Berlin, 2003).

[37] A. Acín, T. Fritz, A. Leverrier, and A. B. Sainz, A combinatorial approach to nonlocality and contextuality, Comm. Math. Phys. 334, 533 (2015).

[38] R. Ramanathan, J. Tuziemski, M. Horodecki, and P. Horodecki, No Quantum Realization of Extremal No-Signaling Boxes, Phys. Rev. Lett. 117, 050401 (2016).
[39] D. E. Knuth, The sandwich theorem, Electron. J. Combin. 1, A1 (1994).

[40] A. Schrijver, Combinatorial Optimization. Polyhedra and Efficiency, Vol. B, Series volume 24 of Algorithms and Combinatorics, Matroids, Trees, Stable Sets (Springer, Berlin, 2003), Chaps. 39-69.

[41] A. B. Sainz, T. Fritz, R. Augusiak, J. B. Brask, R. Chaves, A. Leverrier, and A. Acín, Exploring the local orthogonality principle, Phys. Rev. A 89, 032117 (2014).

[42] J. Gouveia, P. A. Parrilo, and R. R. Thomas, Theta bodies for polynomial ideals, SIAM J. Optim. 20, 2097 (2010).

[43] M. M. Deza and M. Laurent, Geometry of Cuts and Metrics, Vol. 15 of Algorithms and Combinatorics (Springer, Berlin, 1997).

[44] M. Laurent, and S. Poljak, On the facial structure of the set of correlation matrices, SIAM J. Matrix Analysis Appl. 17, 530 (1996).

[45] C.-K. Li, and B.-S. Tam, A note on extreme correlation matrices, SIAM J. Matrix Analysis Appl. 15, 903 (1994). 